\title{
Optimal design and implementation of a temperature and strain optical transducer using FBGs and fiber taper hybrid structure
}

\author{
A. Quintela, L. Rodriguez, M. I. Barquin, C. Galindez, M.A. Quintela and J. M. Lopez-Higuera \\ Grupo de Ingeniería Fotónica, Universidad de Cantabria, \\ Edificio de I+D+i de Ing. de Telecomunicación, Avda. de los Castros s/n, 39005 Santander, Spain
}

\begin{abstract}
A temperature and strain optical fiber transducer and its optimal design are presented. The hybrid structure is composed of two Fiber Bragg Gratings (FBG) in a fused taper. Using the same phase mask one of the FBG is written outside of the taper, and the other one in the middle of the taper, in the area with constant diameter. The taper diameter and the structure length play a key role on the transducer behaviour. Useful results to optimize the transducer structure design, from a theoretical and experimental investigation, are reported in this paper.
\end{abstract}

Keyword list: FBG optic sensor, fiber taper, strain temperature discrimination, optimization.

\section{INTRODUCTION}

Fiber Bragg gratings (FBG) are probably, in the last years, one of the elements more used in the field of fiber optics sensors[1]. The combination of FBGs and fiber tapers is used to improve the behaviour of the FBG, for example for temperature and strain discrimination[2, 3], for fabrication of chirped FBG[3, 4] and modification of strain responses of a FBG based strain transducer[5]. In this paper results from a systematic study concerning the influence of the transducer's key parameters on the strain sensibility and dynamic range are presented and discussed. Useful conclusions for the optimal design of the structure are presented.

\section{THEORY}

An scheme of the transducer proposed is shown in figure 1. Two FBGs with the same period are written in an adiabatic taper. One of the FBG is written outside the Taper but very close to it, and the other inside the taper, in thesection with constant diameter. The length of the structure, $\mathrm{L}$, is the distance between the two points of the transducer where the force will be applied. This structure can be divided into three different sections, with $\mathrm{L}_{1}, \mathrm{~L}_{2}$ and $\mathrm{L}_{3}$ lengths respectively. The former (Section 1) is really the fiber $(125 \mu \mathrm{m}$ of diameter of a standard telecommunications monomode fiber in our study). The section 2 is integrated by two section of a conical fiber and the last one, section 3 , is formed by a fiber wire with constant diameter in the middle of the fiber taper.

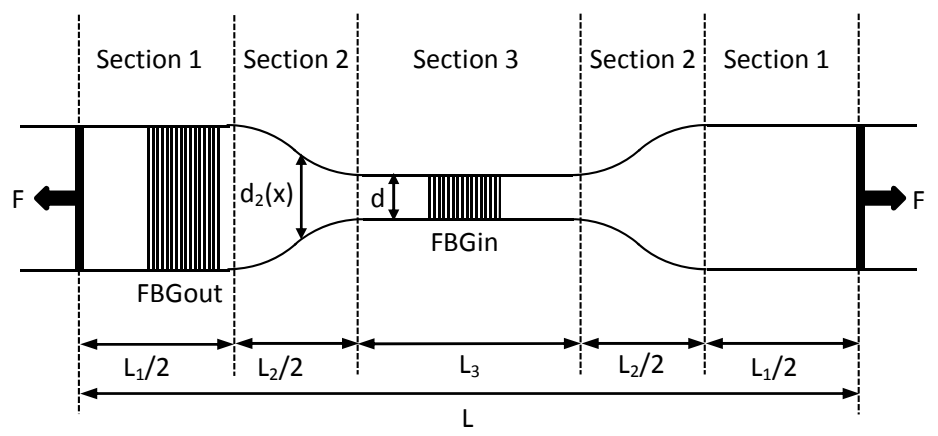

Figure 1. Scheme of the proposed transducer

If a constant longitudinal force is applied to an optic fiber, $\mathrm{F}$, a constant strain, $\varepsilon$, appears along the fiber that is equal to:

$$
\varepsilon=\frac{4 F}{E \pi d^{2}}
$$

21st International Conference on Optical Fiber Sensors, edited by Wojtek J. Bock, Jacques Albert, Xiaoyi Bao, Proc. of SPIE Vol. 7753, 77537G · (C) 2011 SPIE · CCC code: 0277-786X/11/\$18 · doi: 10.1117/12.885966 
where $F$ is the applied force, $d$ is the fiber diameter and $E$ is the Young modulus. So for the case of the proposed structure for a given force, the strain will be different in every section. In the section one and three it will be constant and in the section 2 it will be variable, therefore it would be better to define for this section a medium strain value that is equal to:

$$
\overline{\varepsilon_{2}}=\int \frac{4 F}{E \pi d_{2}(x)^{2}} d x
$$

The relationship between the strains in the three sections in a given taper will be constant and independent of the transducer length. In the case of the section one, $\varepsilon_{1}$, and three, $\varepsilon_{3}$, is given by:

$$
k_{3-1}=\frac{\varepsilon_{3}}{\varepsilon_{1}}=\frac{d_{\text {fiber }}^{2}}{d^{2}}
$$

However the relationship between these strains and the strain of the whole transducer depends on the transducer length and the taper diameter. The strain of the transducer $\left(\varepsilon_{\mathrm{T}}\right)$ could be expressed as:

$$
\varepsilon_{T}=\frac{\Delta L}{L}=\frac{\Delta L_{1}+\Delta L_{2}+\Delta L_{3}}{L_{1}+L_{2}+L_{3}}=\frac{\varepsilon_{1} L_{1}+\overline{\varepsilon_{2}} L_{2}+\varepsilon_{3} L_{3}}{L_{1}+L_{2}+L_{3}}=\frac{\varepsilon_{1}\left(L_{1}+k_{2-1} L_{2}+k_{3-1} L_{3}\right)}{L_{1}+L_{2}+L_{3}}=\varepsilon_{1} \cdot Q
$$

where $Q$ is a value higher than the unity, and $\lim _{L_{1} \rightarrow \infty} Q=1$.

The effective index of the fiber optic depends on its diameter: the smaller the diameter the lower the effective index [6]. So if two FBGs with the same period are written into the same fiber but in sections with different diameter, the Bragg wave length will be small in the FBG wrote in the section with smallest diameter. Obviously, two different Bragg wavelengths are obtained writing with the same phase mask two FBGs in section 1 and 3 respectively.

When a force is applied between the two extreme points of the transducer the strain, and therefore the shifts for the Bragg wavelengths, will be different, being higher for the FBG inside the taper, section 3. Hence, the higher the applied force the shorter the distance between Bragg wavelengths

However a variation of temperature will cause the same shifts in both Bragg wavelengths. The mentioned features drive to the conclusion that the distance between both Bragg wavelengths can be used to establish the transducer strain, and with both absolute positions is possible to obtain the value of temperature.

\section{OPTIMAL DESIGN}

Here, two main features will be optimized with in terms of the two transducer structure parameters. The transducer strain sensitivity (minimum transducer strain increment that it is possible to measure) and the transducer dynamic range (maximum value of strain that it is possible to measure) will be maximize in terms of the taper diameter (d) and the transducer length (L), To change L, only $\mathrm{L}_{1}$ will be modified. First, the two mentioned features will be studied in function of the taper diameter (for a given $\mathrm{L}_{1}$ value).

The value of the dynamic range is given by the maximum value of transducer strain allowing the distance between the two Bragg wavelengths to be detected. The smaller the taper diameter, the greater the distance between both peaks. However this will also imply a higher strain difference in both FBGs, see (3). It should also be taken into account that a higher strain difference will also give rise to a faster convergence of both peaks. In order to find the taper diameter that maximizes it, the dynamic range of the FBG written in the section 1 and not the dynamic range of the transducer will be used really. The reason lies in the fact that both values are proportional being always higher the one of the transducer (4). So for the optimization is the same to use one or the other. However the strain dynamic range in this FBG is independent of the transducer length, and can be defined as the small limit of the transducer dynamic range.

Knowing the effective refractive index dependence with the fiber diameter[6], fixing the minimum peaks distance for example to $0,4 \mathrm{~nm}$ it is possible to express the dynamic range of the $\mathrm{FBG}$ in the section $1\left(\mathrm{DR}_{\mathrm{FBG} 1}\right)$ as:

$$
D R_{F B G 1}=\frac{\lambda_{\text {Bout }}-\lambda_{\text {Bin }}-0.4}{k\left(k_{3-1}-1\right)}=\frac{\lambda_{\text {Bout }}-\lambda_{\text {Bin }}-0.4}{k\left(\frac{125^{2}}{d^{2}}-1\right)}
$$

where $\lambda_{\text {Bout }}$ and $\lambda_{\text {Bin }}$ are the Bragg wavelengths of the FBGs in section 1 and 3 respectively, $k$ is the relationship between strain and wavelengths shift in a FBG, typically $1,1 \mathrm{pm} / \mu \varepsilon$, and $k_{3-1}$ is the relation between the strains in 
section 1 and 3 (3). The obtained results are synthetized in figure $2 \mathrm{a}$. A maximum $\mathrm{DR}_{\mathrm{FBG} 1}$ is obtained for a taper diameter around $100 \mu \mathrm{m}$ considering a $0.4 \mathrm{~nm}$ of minimum peaks distance.

The transducer strain sensitivity here is defined as the variation between both Bragg wavelengths when the transducer strain is incremented in $1 \mu \varepsilon$. The variation of the difference between both peaks in terms of the strain in section 1 , $\varepsilon_{1}$, can be writen as:

$$
\Delta\left(\lambda_{\text {out }}-\lambda_{\text {in }}\right)=k\left(k_{3-1}-1\right)
$$

where $\lambda_{\text {out }}$ is the wavelength of the FBG in section $1, \lambda_{\text {in }}$ is the wavelength of the FBG in section 3 ,. In figure $2 b$ the variation of the transducer sensitivity, with the diameter is shown. As expected, the sensitivity is higher as the taper diameter decreases.

If the taper diameter is fixed, the values of both parameters (dynamic range and sensitivity) will change modifying the value of the transducer length. When $\mathrm{L}$ is increased (increasing L1) the value of $\varepsilon_{1}$ and $\varepsilon_{\mathrm{T}}$ tend to be equal, therefore the transducer dynamic range decreases. On the other hand, when L1 increases, the value of $\varepsilon_{1}$ and $\varepsilon_{3}$ increases too, (3) and (4). So, for a given strain transducer, the distance between both Bragg wavelengths will be higher, and so the transducer sensibility increases.


Figure 2. a) Dynamic strain range of FBG1 vs taper diameter. b) transducer strain sensitivity vs taper diameter.

\section{EXPERIMENTAL RESULTS AND DISCUSSION}

In order to experimentally check the theoretical results obtained in the previous section several FBGs were written outside and inside tapers with different smallest diameters $(61,72,81,96$ and $101 \mu \mathrm{m})$. The tapers were made using a machine developed in the Photonics Engineering Group of the University of Cantabria. The machine uses a mobile oxybutane flame. Their position is computer controlled and using the equations presented in [7] it is possible to obtain adiabatic tapers with a section of constant diameter. In this case the length of this section is $10 \mathrm{~mm}$ to ease the writing process of the FBGs. The diameter of the tapers was characterized using a microscope. The tapers were hydrogen loaded at $125 \mathrm{~atm}$ during one week in order to improve their photosensitivity. In every taper one FBG was written in the section of smallest and constant diameter and another one outside the taper and very close to it (a few mm). All FBGs were written using the same phase mask and a CW argon doubled UV laser $(244 \mathrm{~nm})$. The reflectivity and length for all of them were around $80 \%$ and $4 \mathrm{~mm}$. The separation between the two Bragg wavelengths was from $4,2 \mathrm{~nm}$ for the $50 \mu \mathrm{m}$ taper to $0,7 \mathrm{~nm}$ for the $105 \mu \mathrm{m}$.

Every structure composed by two FBG and a taper were characterised in temperature using a Peltier cell excited by a temperature diode controller equipment. In every case the responses to the temperature were identical.

After that, every structure was characterised in strain, using a computer controlled linear translation stage, for different structure lengths $(78,100,143$ and $168 \mathrm{~mm})$. In figure 3a different results obtained for different lengths for a structure with $61 \mu \mathrm{m}$ diameter taper and a FBG in a fiber without taper, that it is used as reference are shown. In the graphic it can be observed that independently of the length the structure, the relation between the strains in the two transducer FBGs is the same in all cases, as is expressed in (3). From this relation the taper diameter is obtained. In this case the diameter obtained was $62 \mu \mathrm{m}$ very similar to the value obtained from the microscope characterization. For all tapers the values of the diameter obtained from the microscope and the strain measurements were very similar. It was checked that if the 
length of the structure increases, the strain in the FBG outside the taper is more similar to the strain in a fiber without taper, as expressed on equation (4) Another example of the measurements it is shown in figure $3 \mathrm{~b}$ where the value of the wavelength shift for the FBGs of four transducers with different taper diameters and similar transducer length $(168 \mathrm{~mm})$, and a FBG in a fiber without taper are presented.
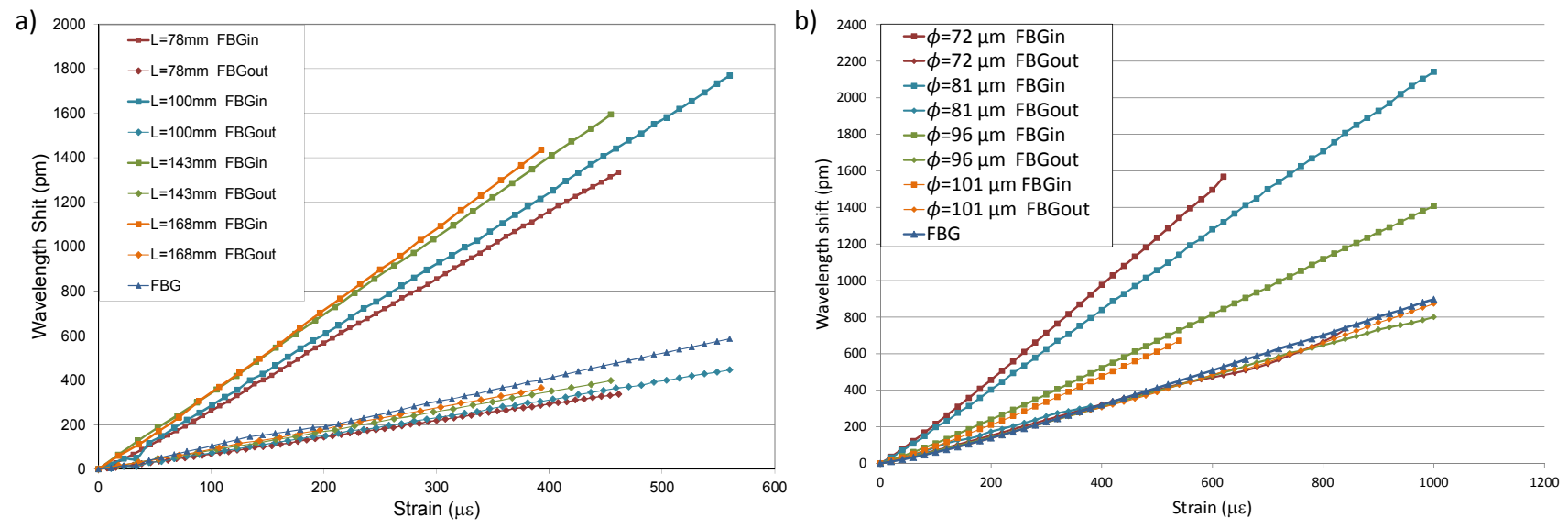

Figure 3. a) FBG wavelength shifts of a $62 \mu \mathrm{m}$ transducer taper diameter for different transducer lengths. b) FBG wavelength shifts of several transducers with a transducer length of $150 \mathrm{~mm}$ and with different taper diameters.

\section{CONCLUSIONS}

A temperature and strain transducer based on a structure composed by two FBG with the same period and an adiabatic taper is theoretically and experimentally studied in this paper. Both FBGs are written in sections with constant diameter, one outside the taper and the other one inside the taper. When a force between both transducer extremes is applied the strain in both FBGs, and the global transducer strain are different but proportional. As the temperature response in both FBGs is similar, it is possible to simultaneously measure strain and temperature. In the design and implementation of the transducer there are two key parameters to optimize the response of the transducer (strain dynamic range, transducer strain sensitivity, etc). For a given fiber a diameter that maximizes both strain dynamic range and strain sensitivity can be found. Both response parameters can be modified to change the transducer length. The higher the transducer length, the smaller the strain dynamic range, resulting in an increase of the strain sensitivity. The theoretical results have been verified. To do that, several hybrid FBGs-fiber taper structures were fabricated and properly experimentally characterized.

\section{ACKNOWLEDGEMENTS}

The authors would like to acknowledge the support provided by the Spanish CICYT through the TEC2007-67987-C0201 and TEC2010-20224-C02-02 projects.

\section{REFERENCES}

[1] J. M. Lopez-Higuera, [Handbook of optical fibre sensing technology] John Wiley \& Sons Inc, (2002).

[2] M. G. Xu, L. Dong, L. Reekie, J. A. Tucknott, and J. L. Cruz, "Temperature-independent strain sensor using a chirped Bragg grating in a tapered optical fibre," Electronics Letters, 31(10), 823-825 (2002).

[3] O. Frazão, M. Melo, P. V. S. Marques, and J. L. Santos, "Chirped Bragg grating fabricated in fused fibre taper for strain-temperature discrimination," Measurement Science and Technology, 16, 984 (2005).

[4] J. M. Lazaro, A. Quintela, W. Urbanczyk, J. Wojcik, and J. M. Lopez-Higuera, "Bragg Gratings Written in Tapered Solid-Core Photonic Crystal Fibers," Photonics Technology Letters, IEEE, 22(14), 1048-1050 (2010).

[5] O. Frazão, S. F. O. Silva, A. Guerreiro, J. L. Santos, L. A. Ferreira, and F. M. Araújo, "Strain sensitivity control of fiber Bragg grating structures with fused tapers," Applied Optics, 46(36), 8578-8582 (2007).

[6] K. C. Byron, K. Sugden, T. Bricheno, and I. Bennion, "Fabrication of chirped Bragg gratings in photosensitive fiber," Electronics Letters, 29(18), 1659-1660 (1993).

[7] J. D. Love, W. M. Henry, W. J. Stewart, R. J. Black, S. Lacroix, and F. Gonthier, "Tapered single-mode fibres and devices. I. Adiabaticity criteria,” IEE Proceedings J Optoelectronics, 138(5), 343-354 (1991). 